\title{
Extending Bounded Holomorphic Functions from Certain Subvarieties of a Strongly Pseudoconvex Domain
}

\author{
Kenzō ADACHI*
}

1. Introduction. G. M. Henkin ([2]) proved that any bounded holomorphic function defined on an analytic closed submanifold in general position in a strongly pseudoconvex domain can be continued to a bounded holomorphic function in the entire domain. In this paper we extend G. M. Henkin's results to certain subvarieties, which possess sufficiently restricted singular points, in a strongly pseudoconvex domain. Our theorem will be proved by applying the integral formula constructed by E. L. Stout ([3]) for certain hypersurfaces in a strongly pseudoconvex domain and the method of G. M. Henkin ([2]) which was used to prove the extension theorem for analytic submanifolds in a strongly convex domain.

2. Let $\Omega$ be an arbitrary subset in $C^{N+1}$. We let $O(\Omega)$ designate the space of holomorphic functions in $\Omega$ and we let $H^{\infty}(\Omega)$ designate the space of bounded holomorphic functions in $\Omega$. Let $D, \tilde{D}(\bar{D} \subset \tilde{D})$ be strongly pseudoconvex domains in $C^{N+1}$ with $C^{\infty}$-boundaries. We fix a function $F \in O(\overline{\tilde{D}}), F \neq 0$. We set $Z(F)$ $=\{z: F(z)=0\}, \Delta=Z(F) \cap D$ and $\bar{\Delta}=Z(F) \cap \tilde{D}$. Then $D$ can be written as $D$ $=\{z \in \tilde{D}: \rho(z)<0\}$, where $\rho$ is a $C^{\infty}$-strongly plurisubharmonic function on $\tilde{D}$ with $d \rho \neq 0$ on $\partial D$. Let $D_{v}=\left\{z \in D: \rho(z)<-\varepsilon_{v}\right\}$ and $\Delta_{v}=Z(F) \cap D_{v}$, where $\left\{\varepsilon_{v}\right\}$ is a sequence of sufficiently small strictly decreasing positive numbers which converges 0 . Now we make the following assumptions:

a) $\Delta$ is a non-empty connected set.

b) $d F \neq 0$ on $Z(F) \cap \partial D$.

c) $Z(F)$ meets $\partial D$ transversally.

In this setting, we have the following formula.

Theorem 1. If $f \in H^{\infty}(\Delta)$, then the following formula holds for all $z \in \Delta$.

$$
f(z)=\lim _{v \rightarrow \infty} \int_{\partial \Delta_{v}} f(\zeta) \frac{\tilde{\Psi}_{z} \tilde{\omega}_{F}}{G(z, \zeta)^{N}\|\operatorname{grad} F(\zeta)\|}=\lim _{v \rightarrow \infty} \int_{\partial \Delta v} f(\zeta) \tilde{K}(z, \zeta), \text { say, }
$$

where $G(z, \zeta)$ is a $C^{\infty}$-function in a neighbourhood $U \times V$ of $\bar{D} \times \partial D$ such that for

Received December 26, 1975.

* Department of Mathematics, Faculty of Science, Ibaraki University, Mito, Japan. 
each $\zeta \in V, G(\cdot, \zeta)$ is holomorphic in $U$ and such that $G(z, \zeta)=0$ implies $z=\zeta$. Moreover, $G$ admits a division

$$
G(z, \zeta)=\sum_{j=1}^{N+1}\left(z_{j}-\zeta_{j}\right) g_{j}(z, \zeta)
$$

with $g_{j}: U \times V \rightarrow C$ of class $C^{\infty}$ and holomorphic in the first variable. $\tilde{\Psi}_{z}(\zeta)$ is a $C^{\infty}(0, N-1)$ form in a neighbourhood of $\bar{D} \times \partial D$ and, for each $\zeta$ near $\partial D$, coefficients of $\tilde{\Psi}_{z}(\zeta)$ is holomorphic in $\bar{D}$. $\tilde{\omega}_{F}$ is given by

$$
\tilde{\omega}_{F}=\sum_{j=1}^{N+1}(-1)^{j-1} \bar{F}_{j} d \zeta_{1} \wedge \cdots \wedge \widehat{\wedge \zeta_{j}} \wedge \cdots \wedge d \zeta_{N+1},
$$

where $F_{j}=\partial F / \partial \zeta_{j}, j=1, \ldots, N+1$, and $\backslash$ means to be omitted.

Proof. By theorem II.1 of E. L. Stout ([3]), it follows that

$$
f(z)=\int_{\partial \Delta_{v}} f(\zeta) \tilde{K}(z, \zeta) \quad \text { for } \quad z \in \Delta_{v} .
$$

Therefore theorem 1 follows.

TheOREM 2. Let

$$
H_{v}(z)=\int_{\partial \Delta v} f(\zeta) \tilde{K}(z, \zeta)
$$

and

$$
L(f)(z)=H(z)=\lim _{v \rightarrow \infty} H_{v}(z) .
$$

Then $H(z)$ is holomorphic on $\bar{D}-\partial \Delta$ and $H(z)=f(z)$ for all $z \in \Delta$.

Proof. For $z \in W \Subset D_{v_{0}}$, and $\nu>\mu \geqq v_{0}$, we have

$$
H_{v}(z)-H_{\mu}(z)=\int_{\partial\left(\Delta_{v}-\Delta_{\mu}\right)} f(\zeta) \tilde{K}(z, \zeta)=\int_{\Delta_{v}-\Delta_{\mu}} f(\zeta) \bar{\partial}_{\zeta} \tilde{K}(z, \zeta) .
$$

Since the form $\bar{\partial}_{\zeta} K(z, \zeta)$ is bounded for $\zeta \in \Delta_{v}-\Delta_{\mu}$ and $z \in W$, there exists a constant $K$ with

$$
\left|H_{v}(z)-H_{\mu}(z)\right| \leqq K \sup _{\zeta \in \Delta}|f(\zeta)| \cdot \operatorname{Vol}\left(\Delta_{\nu}-\Delta_{\mu}\right) .
$$

Hence $H_{v}(z)$ converges locally uniformly on $D$. Therefore $H(z)$ is holomorphic in $D$. For $z \in \partial D-\partial \Delta, H_{v}(z)$ is holomorphic in $z$ for sufficiently large $v$. Since $H_{v}(z)$ converges uniformly in a neighborhood of $z$, together with theorem 1 , theorem 2 follows.

3. Since the problem is local, we may assume that $F=z_{N+1}$. Then $H_{v}(z)$ can be written as 


$$
\begin{aligned}
H_{\nu}(z) & =\int_{\partial \Delta_{v}} f(\zeta) \frac{\sum_{m=1}^{N} \Psi_{m}(z, \zeta)}{G(z, \zeta)^{N}} d \bar{\zeta}_{1} \wedge \cdots \wedge \widehat{d \bar{\zeta}_{m}} \wedge \cdots \wedge d \bar{\zeta}_{N} \wedge \omega(\zeta) \\
& =\int_{\partial \Delta_{v}} f(\zeta) \frac{K(z, \zeta)}{G(z, \zeta)^{N}}, \text { say, where } \omega(\zeta)=d \zeta_{1} \wedge \cdots \wedge d \zeta_{N} .
\end{aligned}
$$

LemmA 1. Let $f(\zeta) \in H^{\infty}(\Delta)$. Then any $z \in(\bar{D}-\partial \Delta)$ and any vector $w=\left(w_{1}, \ldots\right.$, $\left.w_{N+1}\right) \neq 0$ satisfy the equation

$$
\begin{aligned}
\left.\frac{d H(z+\lambda w)}{d \lambda}\right|_{\lambda=0}= & \left.\lim _{v \rightarrow \infty} \frac{d H_{v}(z+\lambda w)}{d \lambda}\right|_{\lambda=0}=\lim _{v \rightarrow \infty} \int_{\partial \Delta_{v}} \frac{f(\zeta) \sum_{j=1}^{N+1} \frac{\partial}{\partial z_{j}} K(z, \zeta) w_{j}}{G(z, \zeta)^{N}} \\
& -\lim _{v \rightarrow \infty} \int_{\partial \Delta_{v}} f(\zeta) \frac{N \sum_{j=1}^{N+1} \frac{\partial}{\partial z_{j}} G(z, \zeta) w_{j} K(z, \zeta)}{G(z, \zeta)^{N+1}}
\end{aligned}
$$

Proof. This follows immediately from the formula (1).

4. Let $z^{0} \in \partial \Delta$. Let $i_{0}$ be an index such that for some $\gamma_{0}>0$

$$
\left|\frac{\partial \rho}{\partial z_{i 0}}\left(z^{0}\right)\right| \geqq \gamma_{0} .
$$

Let

$$
S_{z^{0}, \sigma}=\left\{\zeta \in C^{N+1}:\left|\zeta-z^{0}\right|<\sigma\right\},
$$

Let $z \in S_{2^{0}, \sigma^{*}}$ We consider the system of equations for $\zeta^{0}=\left(\zeta_{1}^{0}, \ldots, \zeta_{N+1}^{0}\right)$ of the following form:

$$
\begin{aligned}
& \quad \sum_{i=1}^{N+1} \frac{\partial \rho}{\partial \zeta_{i}}\left(\zeta^{0}\right)\left(\zeta_{i}^{0}-z_{i}\right)=0, \\
& \zeta_{i=z_{i}}^{0} \quad\left(i=1,2, \ldots, N, i \neq i^{0}\right), \\
& \zeta_{N+1}^{0}=0 .
\end{aligned}
$$

LEMma 2 (G. M. Henkin [2]). There exist positive constants $\sigma_{1}, \gamma_{1}$ and $\gamma_{2}, \gamma_{3}\left(\gamma_{1} \leqq 1\right)$, depending only on $D$ and $M$, such that for any $\sigma \leqq \sigma_{1}$ and any $z \in S_{z^{0}, \gamma_{1} \text { o }}$ there exists a unique solution $\zeta^{0}=\zeta^{0}(z)$ of the system (3) which belongs to the set $S_{z^{0}, \sigma} \cap \tilde{\Delta}$. Here the point $\zeta^{0}=\zeta^{\circ}(z)$ has the following properties;

$$
\begin{gathered}
\left|z-\zeta^{0}(z)\right|^{2} \leqq \frac{1}{\gamma_{2}}\left[\rho(z)-\rho\left(\zeta^{0}(z)\right)\right], \\
\left|z-\zeta^{0}(z)\right|^{2} \geqq\left|z_{N+1}\right|^{2} \geqq \gamma_{3}\left[\rho(z)-\rho\left(\zeta^{0}(z)\right)\right], \\
\zeta^{0}(z)=z \quad \text { for any } z \in S_{z^{0}, \gamma_{1} \sigma} \cap \tilde{\Delta} .
\end{gathered}
$$

Proof. Since $D$ is locally biholomorphic to a convex domain in $C^{N+1}$, we can assume $S_{z^{0}, \sigma_{1}}$ is convex. Then lemma follows from lemma 6 of G. M. Henkin ([2]). 
Lemma 3. Let $f(\zeta)$ be a bounded holomorphic function on $\Delta$. Then any point $z^{0} \in \partial \Delta$ and any point $z \in S_{z^{0}, y_{1} \sigma_{1}}$ satisfy the inequality

$$
\left|\frac{d H\left(\zeta^{0}+\lambda\left(z-\zeta^{0}\right)\right)}{d \lambda}\right|_{\lambda=1}\left|\leqq \gamma_{4} \sup _{\zeta \in \Delta}\right| f(\zeta) \mid,
$$

where $\zeta^{0}=\zeta^{0}(z), \gamma_{1}, \sigma_{1}$ are from lemma 2 , and the $\gamma_{4}$ depends only on $D$ and $\Delta$.

Proof. We set $\varepsilon=\left|z_{N+1}\right|$, where $z=\left(z_{1}, \ldots, z_{N+1}\right) \in S_{z^{0}, \nu_{1} \sigma_{1}}$. Then lemma 2 implies the inequalities

$$
\varepsilon \leqq\left|\zeta^{0}(z)-z\right| \leqq\left\{\frac{\rho(z)-\rho\left(\zeta^{0}(z)\right)}{\gamma_{2}}\right\}^{1 / 2} \leqq \frac{\varepsilon}{\left(\gamma_{2} \gamma_{3}\right)^{1 / 2}}
$$

Since $\sum_{i=1}^{N+1} \frac{\partial \rho}{\partial \zeta_{i}}\left(\zeta^{0}\right)\left(\zeta_{i}^{0}-z_{i}\right)=0$, it follows that

$$
\begin{aligned}
& \left|\sum_{i=1}^{N+1} \frac{\partial G(z, \zeta)}{\partial z_{i}}\left(\zeta_{i}^{0}-z_{i}\right)\right|=\left|\sum_{i=1}^{N+1}\left(\frac{\partial G(z, \zeta)}{\partial z_{i}}+2 \frac{\partial \rho}{\partial \zeta_{i}}\left(\zeta^{0}\right)\right)\left(\zeta_{i}^{0}-z_{i}\right)\right| \\
= & \left|\sum_{i=1}^{N+1}\left(\frac{\partial G(z, \zeta)}{\partial z_{i}}-\frac{\partial G\left(z, \zeta^{0}\right)}{\partial z_{i}}+O\left(\left|\zeta^{0}-z\right|\right)\right)\left(\zeta_{i}^{0}-z_{i}\right)\right|
\end{aligned}
$$

$\leqq \gamma_{5} \varepsilon(|\zeta-z|+\varepsilon)$. Here we have used the following equation (H. Grauert-I. Lieb [1]);

$$
\frac{\partial G\left(z, \zeta^{0}\right)}{\partial z_{i}}=2 \frac{\partial \rho}{\partial \zeta_{i}}\left(\zeta^{0}\right)+O\left(\left|\zeta^{0}-z\right|\right)
$$

By the equation (2), we have the following inequalities:

$$
\begin{aligned}
& \left.\left|\frac{d H\left(\zeta^{0}+\lambda\left(z-\zeta^{0}\right)\right)}{d \lambda}\right|_{\lambda=1}|=| \frac{d H\left(z+\lambda\left(z-\zeta^{0}\right)\right)}{d \lambda}\right|_{\lambda=0} \mid \leqq \\
& \lim _{v \rightarrow \infty}\left\{\gamma_{6} \int_{\partial \Delta v} \cap s_{z^{0}, \sigma_{1}} \frac{|f(\zeta)|\left|z-\zeta^{0}\right| d \lambda}{|G(z, \zeta)|^{N}}+\gamma_{7} \int_{\partial \Delta_{v} \cap s_{z}{ }^{0}, \sigma_{1}} \frac{|f(\zeta)| \varepsilon(|\zeta-z|+\varepsilon) d \lambda}{|G(z, \zeta)|^{N+1}}\right\} \\
& \quad+\gamma_{8} \sup _{\zeta \in \Delta}|f(\zeta)| .
\end{aligned}
$$

By a smooth change of coordinates, we have (H. Grauert-I. Lieb [1])

$$
|G(z, \zeta)| \geqq A\left(R^{2}+x_{1}^{\prime \prime}\right), \quad \text { for } \quad \rho(\zeta) \geqq \rho(z),
$$

where $z_{j}-\zeta_{j}=x_{j}^{\prime}+i x_{j}^{\prime \prime}, j=1, \ldots, N+1$, and $|z-\zeta|=R$. If we set

$$
x_{1}^{\prime \prime 2}+x_{2}^{\prime 2}+\cdots+x_{2 N}^{\prime \prime 2}=r^{2} \text {, and } \varepsilon^{2}=x_{2 N+1}^{\prime 2}+x_{2 N+1}^{\prime \prime 2},
$$

then to prove the lemma 3 , it suffices to show the following inequalities:

$$
\begin{aligned}
& I_{1}: \int_{0}^{\pi} \int_{0}^{1} \frac{r^{2 N-2} \sin ^{2 N-3} \phi d \phi d r}{\left(\left(r^{2}+\varepsilon^{2}\right)^{2}+r^{2} \cos ^{2} \phi\right)^{N+1 / 2}} \leqq \gamma_{8} \frac{1}{\varepsilon}, \\
& I_{2}: \int_{0}^{\pi} \int_{0}^{1} \frac{r^{2 N-2}\left(r^{2}+\varepsilon^{2}\right)^{1 / 2} \sin ^{2 N-3} \phi d \phi d r}{\left(\left(r^{2}+\varepsilon^{2}\right)^{2}+r^{2} \cos ^{2} \phi\right)^{N+1 / 2}} \leqq \gamma_{9} \frac{1}{\varepsilon},
\end{aligned}
$$




$$
I_{3}: \int_{0}^{\pi} \int_{0}^{1} \frac{r^{2 N-2} \sin ^{2 N-3} \phi d \phi d r}{\left(\left(r^{2}+\varepsilon^{2}\right)^{2}+r^{2} \cos ^{2} \phi\right)^{N+1 / 2}} \leqq \gamma_{10} \frac{1}{\varepsilon^{2}}
$$

$I_{1}$ and $I_{3}$ are consequences of $I_{2}$. But $I_{2}$ follows immediately by simple calculations. Therefore lemma 3 is proved.

Theorem 3. Under the hypothesis (A), formula (1) defines a bounded linear operator $L: H^{\infty}(\Delta) \rightarrow H^{\infty}(D)$.

Proof. We let $(\partial \Delta)_{\sigma}$ designate the $\sigma$-neighbourhood of the manifold $\partial \Delta$. By G. M. Henkin ([2]), it suffices to show that

$$
\sup _{z \in\left[(\partial \Delta)_{v}-\partial \Delta_{v}\right] \cap \partial D_{v}}|H(z)| \leqq \gamma_{11} \sup _{\zeta \in \Delta}|f(\zeta)|,
$$

where the constant $\gamma_{11}$ depends only on $D$ and $\Delta$. We now fix $z \in\left[\left(\partial \Delta_{v}\right)_{\sigma}-\partial \Delta_{v}\right]$ $\cap \partial D_{v}$. We set $\varepsilon=\left|z_{N+1}\right|$. Then $\varepsilon \leqq \sigma$. Let the point $z^{0} \in \partial \Delta$ be such that $z$ $\in S_{z^{0}, \sigma}$. Let $\sigma<\frac{1}{2} \gamma_{1}^{2} \sigma_{1}$. Then by lemma 2 there exists a solution $\zeta^{0}=\zeta^{0}(z)$ of the system (3) belonging to the set $\left(S_{z^{0}, \nu_{1} \sigma_{1} / 2}\right) \cap \tilde{J}$ and satisfying the inequality

$$
\gamma_{3} \varepsilon^{2} \leqq \rho(z)-\rho\left(\zeta^{0}(z)\right) \leqq \frac{1}{\gamma_{2}} \varepsilon^{2} .
$$

Let

$$
T_{v}=\left\{\lambda \in C: Z(\lambda)=\zeta^{0}+\lambda\left(z-\zeta^{0}\right) \in D_{v} \cap S_{z^{0}, \sigma_{1}}\right\} .
$$

$T_{v}$ is a convex domain containing $\lambda=0$, since $D_{v} \cap S_{\mathbf{z}^{0}, \sigma_{1}}$ is convex. For any $\lambda$ we have

$$
\sum_{i=1}^{N+1} \frac{\partial \rho}{\partial \zeta_{i}}\left(\zeta^{0}\right)\left(\zeta_{i}^{0}-z_{i}(\lambda)\right)=0
$$

From equation (5), we have

$$
\left|\lambda\left(z-\zeta^{0}\right)\right|^{2}=\left|z(\lambda)-\zeta^{0}\right|^{2} \leqq \frac{1}{\gamma_{3}}\left\{\rho(z(\lambda))-\rho\left(\zeta^{0}\right)\right\} .
$$

Hence for $\lambda \in \partial T_{v} \cap \partial D_{v}$ we obtain

$$
\left|z(\lambda)-z^{0}\right| \leqq\left|z(\lambda)-\zeta^{0}\right|+\left|\zeta^{0}-z^{0}\right| \leqq \frac{\sigma}{\left(\gamma_{2} \gamma_{3}\right)^{1 / 2}}+\frac{1}{2} \sigma_{1} \gamma_{1} .
$$

We impose one further restriction on the constant $\sigma: \sigma \leqq \frac{1}{2} \sigma_{1} \gamma_{1}\left(\gamma_{2} \gamma_{3}\right)^{1 / 2}$. Then by the uniqueness of a solution of the system (3), it follows that $\zeta^{0}(z(\lambda))=\zeta^{0}(z)$ $=\zeta^{0}$ for any $\lambda \in \partial T_{v} \cap \partial D_{v}$. We obtain the following inequalities from lemma 2 :

$$
|\lambda| \frac{\varepsilon}{\left(\gamma_{2} \gamma_{3}\right)^{1 / 2}}>|\lambda|\left|\left(z-\zeta^{0}\right)\right|=\left|z(\lambda)-\zeta^{0}\right| \geqq\left(\gamma_{2} \gamma_{3}\right)^{1 / 2} \varepsilon
$$

Consequently 


$$
|\lambda| \geqq \gamma_{2} \gamma_{3} \quad \text { for any } \quad \lambda \in \partial T_{v} \cap \partial D_{v} .
$$

If $\lambda \in \partial T_{v}-\partial D_{v}$, then there exist $\gamma_{12}$ such that $|\lambda| \geqq \gamma_{12}$. Let $\gamma_{13}=\min \left(\gamma_{2} \gamma_{3}, \gamma_{12}\right)$. Then

$$
|\lambda| \geqq \gamma_{13}
$$

for any $\lambda \in \partial T_{v}$. By lemma 3, we have

$$
\left|\frac{d H_{v}\left(\zeta^{0}+t\left(z(\lambda)-\zeta^{0}\right)\right)}{d t}\right|_{t=1}\left|\leqq \gamma_{4} \sup _{\zeta \in \Delta}\right| f(\zeta) \mid
$$

for any $\lambda \in \partial T_{v}$. We note that

$$
\left.\frac{d H_{v}\left(\zeta^{0}+t\left(z(\lambda)-\zeta^{0}\right)\right)}{d t}\right|_{t=1}=\lambda \frac{d H_{v}\left(\zeta^{0}+\lambda\left(z-\zeta^{0}\right)\right)}{d \lambda} .
$$

From (6) and (7) we have

$$
\left|\frac{d H_{v}\left(\zeta^{0}+\lambda\left(z-\zeta^{0}\right)\right)}{d \lambda}\right| \leqq \frac{\gamma_{7}}{|\lambda|} \sup _{\zeta \in \Delta}|f(\zeta)|
$$

for any $\lambda \in \partial T_{v}$. Since the function $d H_{v}\left(\zeta^{0}+\lambda\left(z-\zeta^{0}\right)\right) / d \lambda$ is holomorphic in $\lambda$ for all $\lambda \in \bar{T}_{v}$, it follows that

$$
\sup _{\lambda \in T_{v}}\left|\frac{d H_{v}\left(\zeta^{0}+\lambda\left(z-\zeta^{0}\right)\right)}{d \lambda}\right| \leqq \frac{\gamma_{7}}{\gamma_{13}} \sup _{\zeta \in \Delta}|f(\zeta)|
$$

\section{Consequently}

$$
\left|H_{v}(z)-H_{v}\left(\zeta^{0}\right)\right|=\left|\int_{0}^{1} \frac{d}{d \lambda} H_{v}\left(\zeta^{0}+\lambda\left(z-\zeta^{0}\right)\right) d \lambda\right| \leqq \frac{\gamma_{7}}{\gamma_{13}} \sup _{\zeta \in \Delta}|f(\zeta)| .
$$

It follows from (4) that $\zeta^{0} \in \Delta_{v}$. Since $H_{v}\left(\zeta^{0}\right)=f\left(\zeta^{0}\right)$, we obtain the following inequality

$$
\left|H_{v}(z)\right| \leqq\left(\frac{\gamma_{7}}{\gamma_{13}}+1\right) \sup _{\zeta \in \Delta}|f(\zeta)|
$$

Therefore

$$
|H(z)| \leqq\left(\frac{\gamma_{7}}{\gamma_{13}}+1\right) \sup _{\zeta \in \Delta}|f(\zeta)| .
$$

Therefore theorem 3 is proved. 


\section{References}

[1] H. Grauert and I. Lieb, Das Ramirezsche Integral und die Gleichung $\bar{\partial} f=\alpha$ im Bereich der Beschränkten Formen, Rice University Studies, 56, No. 2 (1970), 29-50.

[2] G. M. Henkin, Continuation of bounded holomorphic functions from submanifolds in general position to strictly pseudoconvex domains, Math. USSR Izvestija, 6, No. 3 (1972), 536-563.

[3] E. L. Stout, An integral formula for holomorphic functions on strictly pseudoconvex hypersurfaces, Duke Math. J., 42, No. 2 (1975), 347-356.

Present address: Department of Mothematics, Faculty of Education, Nagasaki University, Nagasaki, Japan 